\title{
Microbial evaluation of proton-pump inhibitors and the risk of pneumonia
}

\author{
S.C.A. Meijvis*»**, M.C.A. Cornips ${ }^{\#, * *}$, G.P. Voorn ${ }^{\star}$, P.C. Souverein\#, H. Endeman ${ }^{+}$, \\ D.H. Biesma*, H.G.M. Leufkens ${ }^{\#}$ and E.M.W. van de Garde ${ }^{\#, f}$
}

ABSTRACT: Recent initiation of proton-pump inhibitor (PPI) treatment may increase the risk of community-acquired pneumonia (CAP), hypothetically by allowing colonisation of the oropharynx by gastrointestinal bacteria. The aim of this study was to assess the causal pathway by considering microbial aetiology of pneumonia and indications for initiation of PPI treatment.

This was a population-based, case-control study with 430 cases with pneumonia and 1,720 matched controls. An elaborate diagnostic protocol was used to identify the causative microorganism of pneumonia. For patients recently starting PPI treatment, indications for treatment were assessed.

Recent initiation of PPI treatment ( $<30$ days) was associated with an increased risk of CAP (adjusted OR 3.1, 95\% Cl 1.4-7.1). Oropharyngeal bacteria were evenly distributed among current users, past users and nonusers of PPIs $(p=0.41)$. Gastrointestinal bacteria were identified in only five $(1.2 \%)$ patients with pneumonia (two current users and three nonusers). Excluding patients who were possibly prescribed PPI treatment for early symptoms of pneumonia (protopathic bias) did not alter the study findings.

This study reaffirmed that use of PPIs is associated with an increased risk of CAP, especially when treatment has recently been started. Neither protopathic bias nor shifts in microbial aetiology seem to explain the association.

KEYWORDS: Case-control study, community-acquired pneumonia, proton-pump inhibitors

$\mathbf{R}$ ecent evidence has suggested that gastric acid-suppressive medication might increase the risk of community-acquired pneumonia (CAP). Results have been conflicting, however, and a meta-analysis failed to draw a definite conclusion due to significant heterogeneity [1-10]. Some researchers are sceptical about the reported association because causality seemed improbable and results are suspected to be biased [11-13]. Given the widespread use of these medications and the severity of pneumonia, further research is warranted. To date, most studies have used medical record databases to examine the use of proton-pump inhibitors (PPIs) in relation to the incidence of CAP. The shortcomings of this approach are inherent to retrospective epidemiological research on administrative databases. Misclassification of cases might have occurred because clinical information (such as radiographic data) was not always available. Confounding by indication and protopathic bias (when treatment for the first symptoms of a disease appears to cause the disease) could not be ruled out because most databases did not include information on the indication for PPI treatment. Furthermore, there were no conclusive data on the causative organisms of CAP included in these analyses. Such data would provide more insights into often suggested, but not demonstrated, causal mechanisms, namely overgrowth and microaspiration of gastrointestinal bacteria.

In the present study, we attempted to overcome the methodological limitations addressed above by including a well-defined cohort of hospitalised CAP patients with elaborate clinical and microbial information, and matching them to a population-based control group.

The aim of the present study was to examine the association between the use of PPIs and CAP by including microbial aetiology and clinical characteristics of patients with pneumonia who recently started PPI treatment in the analyses.

\section{METHODS}

\section{Study design}

This was a population-based, matched casecontrol study where cases were defined as patients with CAP admitted to the St Antonius
AFFILIATIONS

${ }^{*}$ Depts of Internal Medicine,

"Medical Microbiology and

Immunology,

${ }^{f}$ Clinical Pharmacy, St Antonius

Hospital, Nieuwegein,

"Division of Pharmacoepidemiology and Clinical Pharmacology, Utrecht

University, and

+Dept of Intensive Care Medicine,

Diakonessenhuis, The Netherlands.

**These authors contributed equally

to the study.

CORRESPONDENCE

S.C.A. Meijvis

Dept of Internal Medicine

St Antonius Hospital

Postbus 2500

3430 EM Nieuwegein

The Netherlands

E-mail:s.meijvis@

antoniusziekenhuis.nl

Received:

Feb 042011

Accepted after revision:

March 052011

First published online:

April 082011 
Hospital (Nieuwegein, the Netherlands) or the Gelderse Vallei Hospital (Ede, the Netherlands), both of which are teaching hospitals ( 880 and 500 beds, respectively). Population controls were drawn from the PHARMO Record Linkage System database. The PHARMO Institute (Utrecht, the Netherlands) is an independent scientific research organisation studying drug use and outcomes. Their records include detailed information on patient demographics, drug use and hospital admissions, and $\sim 3$ million community-dwelling inhabitants of 48 geodemographic areas in the Netherlands are included $[14,15]$.

\section{Cases}

Cases were patients with confirmed pneumonia who participated in two clinical trials $[16,17]$. Consecutive patients were included if they presented to the emergency department between October 2004 and August 2006, and between November 2007 and February 2010. Pneumonia was defined as a new infiltrate on a chest radiograph plus at least two of the following criteria: cough; sputum production; temperature $>38^{\circ} \mathrm{C}$ or $\angle 35.5^{\circ} \mathrm{C}$; auscultatory findings consistent with pneumonia, leukocytosis or leukopenia $\left(>1 \times 10^{7}\right.$ cells $\cdot \mathrm{L}^{-1},<4 \times 10^{6}$ cells $\cdot \mathrm{L}^{-1}$ or $>10 \%$ rods in leukocyte differentiation); and C-reactive protein more than three times the upper limit of normal. Patients who were immunocompromised (haematological malignancies or immunosuppressive therapy, including the use of $>20 \mathrm{mg}$ prednisone equivalent per day for $>3$ days) were excluded. The study was approved by the local Medical Ethics Committee (St Antonius Hospital) and all patients gave their written informed consent. On the day of hospital admission, pneumonia severity index (PSI) was calculated [18]. Need for intensive care unit (ICU) admission and in-hospital mortality were assessed.

\section{Controls}

Control subjects were obtained from the PHARMO database and individually matched by year of birth, sex and index date to the cases in a 4:1 ratio. The index date was the date of the CAP diagnosis of the corresponding case. Controls with a hospitalisation for CAP during the research period (i.e. in the 6 months before index date, identified by the International Classification of Diseases, 9th edition) were excluded.

\section{Pathogen identification}

The diagnostic tools used to identify the causative microorganism of CAP have been described previously [16]. Briefly, at least two sets of separate blood and sputum samples were cultured from each patient. Sputum was analysed using an inhouse PCR for atypical pathogens (Mycoplasma pneumoniae, Legionella pneumophila, Coxiella burnetii, Chlamydophila pneumoniae and Chlamydophila psittaci). Urine was sampled for antigen testing of Streptococcus pneumoniae and L. pneumophila serogroup 1. In addition, serum samples taken on the day of hospital admission and day 10 were analysed in pairs for detection of a four-fold rise in antibodies to respiratory viruses, C. burnetii, M. pneumoniae, and C. psittaci by complement fixation assay. In addition, antibodies against pneumococcal polysaccharides of 14 different serotypes were measured using the Luminex $x \mathrm{MAP}_{\mathbb{B}}$ Pneumococcal Immunity Panel (Luminex, Oosterhout, the Netherlands) [19]. Pharyngeal samples were obtained for viral culture. Pathogens were classified in two different ways. First, S. pneumoniae, Haemophilus influenzae, Staphylococcus aureus, $H$. parainfluenzae and other streptococci were considered oropharyngeal bacteria. Secondly, pathogens Escherichia coli and Klebsiella pneumoniae were considered gastrointestinal bacteria.

\section{Exposure definition}

Community pharmacies were approached in order to identify all dispensed prescription drugs for cases issued in the 6 months before CAP diagnosis. For controls, drug dispensing records were retrieved from the PHARMO database. Exposure definition was identical for cases and controls. PPIs were not available over the counter in the Netherlands during the study period. We identified all prescriptions for omeprazole, pantoprazole, lansoprazole, rabeprazole and esomeprazole for both cases and controls. Current use of a PPI was defined as a dispensed prescription that lasted beyond 30 days before the index date or started after 30 days before the index date. Past use of a PPI was defined as one or more dispensed prescriptions in the 6 months before the index date that did not last beyond 30 days before index date. Nonuse was defined as no dispensed prescriptions during the 6-month period. These categories were mutually exclusive for each category. A subdivision of the group of current users was made according to the date of the first prescription. Recent initiation was defined as a first prescription $<30$ days before the index date; chronic use was defined as a first prescription $\geqslant 30$ days before index date. Defined daily doses were calculated based on strength and prescribed dosing regimen of the most recent prescription prior to the index date to express the prescribed daily dose within current users [20]. For all patients who started PPI treatment within 15 days prior to the index date, the indications for starting treatment were assessed by telephone interview with the patient and or the prescribing physician.

\section{Potential confounders}

Current use of statins, angiotensin-converting enzyme inhibitors and angiotensin II receptor antagonists was defined analogously to current PPI use. These drugs have been reported to influence the risk of CAP [8]. Exposure to the following medications was used as a proxy (indicator for disease) for comorbid illness predisposing to CAP and was defined as two or more prescriptions in the 6 months before the index date. We evaluated use of nonsteroidal antiinflammatory drugs (NSAIDs), antidiabetics (as a proxy for diabetes mellitus (DM)), opiates, antiplatelet therapy, inhalation medication (as a proxy for chronic obstructive pulmonary disease (COPD) or asthma) and digoxin plus diuretics (as a proxy for congestive heart failure (CHF)). Besides these, inhaled corticosteroids and anticholinergics were also evaluated as separate potential confounders [21, 22]. Prescriptions for oral corticosteroids during the month prior to the index date and for antibiotics during the 6 months prior to the the index date were also assessed.

The sensitivity of the proxies for DM, COPD or asthma, and CHF was checked by studying the consistency of the proxy with the corresponding disease, as recorded in the medical charts of cases.

\section{Statistical analysis}

Conditional logistic regression analysis was performed to obtain crude odds ratios (ORs) in matched cases and controls. 
Data are presented as $\mathrm{n}(\%)$, OR $(95 \% \mathrm{CI})$ or p-values. We considered factors associated with CAP in the univariate analysis and variables previously found to be associated with CAP and PPI use as potential confounders in the multivariate model. We selected potential confounders for the multivariate model stepwise by direct estimation of the degree of confounding produced by each variable (relative change in OR for CAP associated with current use of PPI). We continued including potential confounders in the multivariate model until further addition of confounders modified the OR by $<5 \%$.

A backward logistic regression analysis including age, sex, comorbidities (CHF, COPD or asthma, DM and renal failure) and PSI score was used to study the outcome of CAP in relation to the use of PPIs. The association between PPI use and causative agents of CAP was studied using Chi-squared and Fisher's exact tests where appropriate.

\section{RESULTS}

\section{Characteristics of cases and controls}

The study population comprised $430 \mathrm{CAP}$ cases and 1,720 matched controls. Characteristics of cases and controls are shown in table 1 . The mean $\pm \mathrm{SD}$ age of cases and controls was $62 \pm 18$ yrs and $59 \%$ were male. Among cases, 32 patients were admitted to the ICU and 24 patients died during their hospital stay. Overall, cases were more likely to use medication than controls.

\section{Association between use of PPIs and CAP}

Table 2 lists the crude and adjusted ORs for CAP associated with use of PPIs. In the crude analysis, current use of PPIs was associated with an OR for CAP of 1.8 (95\% CI 1.4-2.4). In the final multivariate model, oral corticosteroids, inhaled corticosteroids, anticholinergics and NSAIDs were included as confounders. The adjusted OR for CAP associated with current PPI use was 1.6 (95\% CI 1.2-2.2).

The risk of CAP increased as the starting date of the PPI approached the index date. To ensure that patients identified as new users were not intermittent users, only cases and controls that had not redeemed a prescription for PPIs during the year before the index date were included. Patients with a first prescription $\leqslant 15$ days before the index date had an adjusted OR of 3.1 (95\% CI 1.1-8.8). Patients with a first prescription 16-29 days before the index date had an adjusted OR of 3.3 (95\% CI 0.91-11.6). A sensitivity analysis including new users who received PPIs during the 0.5 -yr period of 12 up to 6 months before the index date (but did not receive any prescriptions from $>6$ months before the index date until $<30$ days before the index date), included one new user for cases and six new users for controls. In this analysis, recent initiation of PPI treatment remained significantly associated with an increased risk for CAP (adjusted OR 2.4, 95\% CI 1.15.0). As shown in table 2 , there was a modest dose-effect relationship for current use of PPIs.

\section{Clinical details of CAP cases recently starting PPI treatment}

Table 3 provides clinical background information on the pneumonia patients that recently started PPI treatment. Medical history differed markedly between patients, although cardiovascular disease and COPD were common comorbidities. The indications for PPI treatment were diverse as well. Patient 7 received a PPI for xyphoid pain, which might have been CAP-, and not reflux-, related. Patient 9 also experienced possible symptoms of CAP. Half of the patients received a PPI as prophylaxis for gastrointestinal bleeding and ulcers due to NSAIDs. There was no reason to suspect that their pain complaints (e.g. lower back pain) were early symptoms for

TABLE 1 Characteristics of community-acquired pneumonia cases and controls

\begin{tabular}{|c|c|c|c|c|}
\hline Subjects & 2150 & 430 & 1720 & \\
\hline Males & $1270(59)$ & $254(59)$ & $1016(59)$ & NA \\
\hline ACE inhibitors & $343(16)$ & $80(19)$ & $263(15)$ & $1.3(0.99-1.8)$ \\
\hline Angiotensin receptor antagonists & $210(9.8)$ & $32(7.4)$ & $178(10)$ & $0.69(0.46-1.0)$ \\
\hline COPD or asthma drugs ${ }^{\#}$ & $276(13)$ & $111(26)$ & $165(9.6)$ & $3.4(2.6-4.5)$ \\
\hline Inhaled corticosteroids ${ }^{\#}$ & $234(11)$ & $86(20)$ & $148(8.6)$ & $2.7(2.0-3.6)$ \\
\hline Anticholinergics ${ }^{\#}$ & $169(7.9)$ & $69(16)$ & $100(5.8)$ & $3.3(2.4-4.7)$ \\
\hline No inhalation steroids or anticholinergics & $36(1.7)$ & $5(1.2)$ & $31(1.8)$ & $0.64(0.25-1.7)$ \\
\hline CHF medication & $50(2.3)$ & $19(4.4)$ & $31(1.8)$ & $2.5(1.4-4.6)$ \\
\hline Opiates & $125(5.8)$ & $21(4.9)$ & $104(6.0)$ & $0.80(0.49-1.3)$ \\
\hline
\end{tabular}

Data are presented as $n$, mean \pm SD or $n(\%)$, unless otherwise stated. ACE: angiotensin-converting enzyme; COPD: chronic obstructive pulmonary disease; CHF: congestive heart failure; NSAID: nonsteroidal anti-inflammatory drug; NA: not applicable. ${ }^{*}$ : not mutually exclusive categories. 


\begin{tabular}{|c|c|c|c|c|c|c|c|}
\hline Nonuser & $1690(79)$ & $307(71)$ & $1383(80)$ & Reference & & Reference & \\
\hline Past user & $90(4.2)$ & $20(4.7)$ & $70(4.1)$ & $1.3(0.78-2.2)$ & 0.308 & $1.2(0.72-2.1)$ & 0.46 \\
\hline Current user & $370(17)$ & $103(24)$ & 267 (16) & $1.8(1.4-2.4)$ & $<0.01$ & $1.6(1.2-2.2)$ & $<0.01$ \\
\hline Recent $^{+}$ & $28(7.6)$ & $12(12)$ & $16(6.0)$ & $3.4(1.6-7.3)$ & $<0.01$ & $3.1(1.4-7.1)$ & $<0.01$ \\
\hline $0-15$ days & $16(4.3)$ & $7(6.8)$ & $9(3.4)$ & $3.5(1.3-9.6)$ & 0.012 & $3.1(1.1-8.8)$ & 0.04 \\
\hline 16-29 days & $12(3.2)$ & $5(4.9)$ & $7(2.6)$ & $3.3(1.1-10.4)$ & 0.044 & $3.3(0.91-11.6)$ & 0.07 \\
\hline Chronic $^{\S}$ & $342(92)$ & $91(88)$ & 251 (94) & $1.7(1.3-2.3)$ & $<0.01$ & $1.5(1.1-2.1)$ & $<0.01$ \\
\hline \multicolumn{8}{|l|}{$\mathbf{D D D s}^{f}$} \\
\hline$<1.5$ & $292(79)$ & $80(78)$ & $212(79)$ & $1.8(1.3-2.4)$ & $<0.01$ & $1.6(1.2-2.2)$ & $<0.01$ \\
\hline
\end{tabular}

Data are presented as $n$ or $n(\%)$, unless otherwise stated. DDD: daily defined dose. *: adjusted for use of inhalation corticosteroids, anticholinergics, nonsteroidal antiinflammatory drugs and oral corticosteroids; ${ }^{\top}$ : days from first prescription until index date; ${ }^{+}:<30$ days; ${ }^{\varsigma}: \geqslant 30$ days; ${ }^{f}$ : analysis within current users.

CAP. The indications for the remaining cases were (bleeding) ulcers, Helicobacter pylori infection and dyspepsia.

In order to assess whether protopathic bias could explain the demonstrated increase in risk associated with recent initiation of PPI treatment, we conducted a sensitivity analysis by considering cases 7 and 9 as nonexposed. The risk for CAP remained significantly elevated for recent initiation of PPI treatment (adjusted OR 2.5, 95\% CI 1.0-5.8). If cases 1, 7 and 9 were excluded (because prescriptions were issued $\leqslant 2$ days before CAP diagnosis, which could be too short to produce an acid-suppressive effect and subsequent change in commensal flora) the adjusted OR was 2.1 (95\% CI 0.85-5.1).

\section{Clinical outcomes}

Four (33\%) out of the 12 patients who recently started PPI treatment ( $<30$ days before admission) were admitted to the ICU, whereas only 7 and $11 \%$ of nonusers and current users, respectively, were admitted to the ICU. After adjusting for comorbidities, age, sex and PSI score, recent initiation of PPI treatment was independently associated with ICU admission $(\mathrm{p}<0.01)$. Chronic and past use were not associated with ICU admission $(p=0.89$ and $p=0.99$, respectively). None of the patients who recently started PPI treatment died during their hospital stay.

\section{Causative pathogens}

Among CAP patients, S. pneumoniae was identified in 30\% of cases. In $36 \%$ of cases, a causative organism could not be identified. Table 4 shows the microbial aetiology for current users, past users and nonusers of PPIs.

Of the 430 CAP cases, five were caused by defined gastrointestinal bacteria. Three (1\%) of these were not receiving PPI treatment and two (2\%) were current users of PPIs. Defined oropharyngeal bacteria were identified in $41 \%$ of current users, $25 \%$ of past users and $39 \%$ of nonusers ( $\mathrm{p}=0.41$ versus nonoropharyngeal and unidentified pathogens). The frequency of oropharyngeal pathogens did not differ between patients recently starting PPI treatment and nonusers $(\mathrm{p}=1.00$ versus nonoropharyngeal and unidentified pathogens).

\section{Performance of proxies in cases}

The results of the comparison of our proxies with recorded medical diagnoses are shown in figure 1.

\section{DISCUSSION}

In this study, the risk of CAP was increased in patients currently using a PPI. We confirmed that the risk was highest shortly after initiation of PPI treatment. Because of this seemingly contradictory timing effect, we further examined the CAP patients who recently started PPI treatment. It became clear that protopathic bias is not the sole explanation for the observed risk. Study of the causative microorganism of CAP did not show an increase in the frequency of either oropharyngeal or gastrointestinal bacteria in patients using PPIs.

LAHEIJ et al. [7] were the first to report a positive association between current use of PPIs and risk of CAP. Most, but not all subsequent studies confirmed this association and also described a gradual increase in effect size when treatment was started closer to the index date. As maximum acid suppression is reached after 7 days of PPI treatment, this pattern of association is difficult to account for. Protopathic bias has been put forward as a possible explanation: patients presenting with CAP-related cough might be misdiagnosed as having gastro-oesophageal reflux disease or patients presenting with CAP-related pain might be prescribed an NSAID with a PPI for prophylaxis. Our study is the first to provide detailed information on patients who recently started PPI treatment, for whom the supposed association is most controversial. Only two of these patients received a prescription intended for complaints that might have been linked to early pneumonia, and a sensitivity analysis excluding these cases showed that the observed association remained increased.

Previous reports have suggested that backflow and overgrowth of gastrointestinal bacteria during PPI treatment may result in colonisation of the oral space and predispose to 


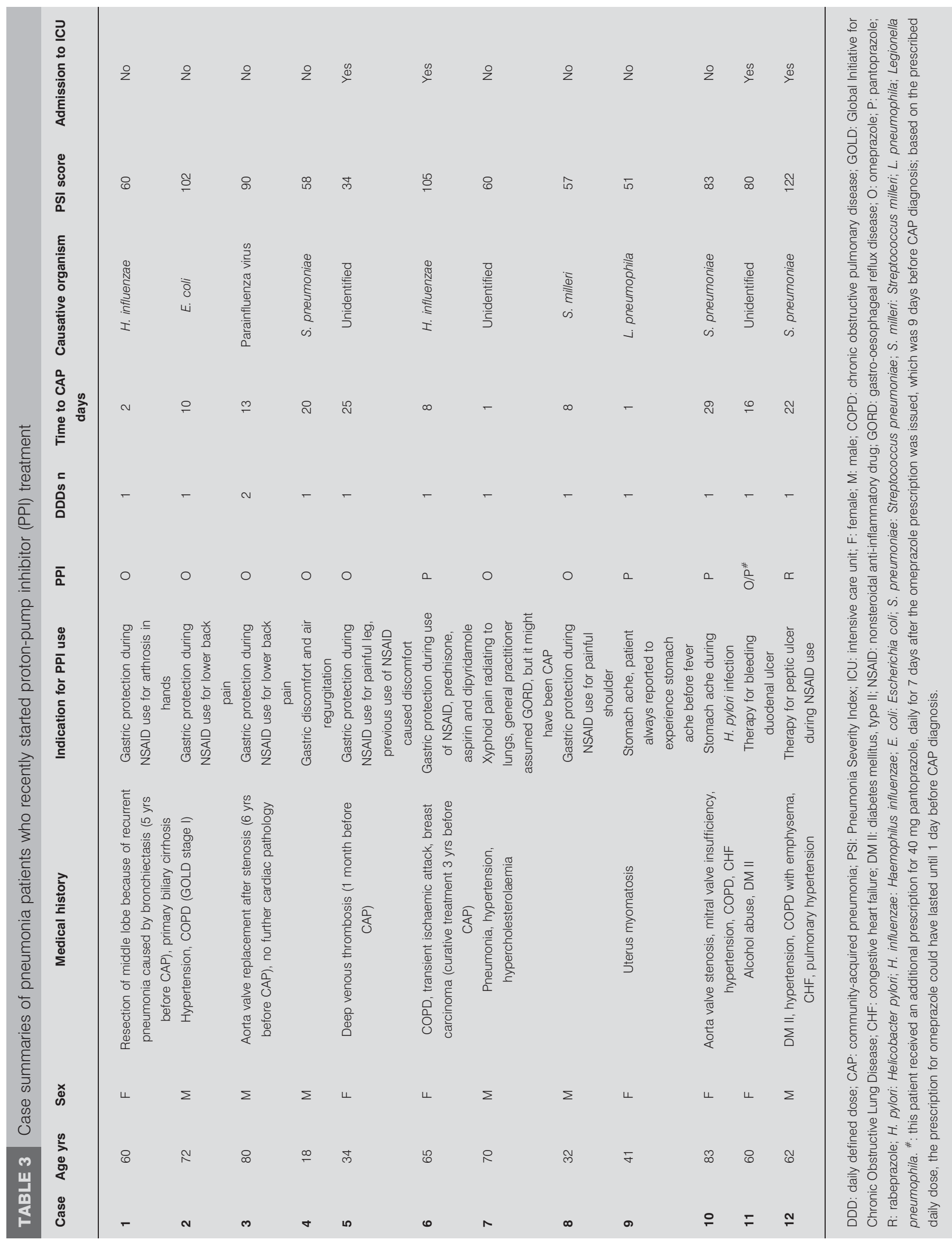




\begin{tabular}{|c|c|c|c|c|}
\hline & All & Nonusers & Current users & Past users \\
\hline Subjects & 430 & 307 & 103 & 20 \\
\hline S. pneumoniae & $130(30)$ & $97(32)$ & $28(27)$ & $5(25)$ \\
\hline Atypical & $69(16)$ & $54(18)$ & $7(6.8)$ & $8(40)$ \\
\hline Other & $15(3.5)$ & $9(2.9)$ & $6(5.8)$ & $0(0)$ \\
\hline Unidentified & $154(36)$ & $103(34)$ & $44(43)$ & $7(35)$ \\
\hline Oropharyngeal bacteria identified ${ }^{\#}$ & $166(39)$ & $119(39)$ & $42(41)$ & $5(25)$ \\
\hline Gastrointestinal bacteria identified & $5(1.2)$ & $3(1.0)$ & $2(1.9)$ & $0(0)$ \\
\hline
\end{tabular}

Data are presented as n or $\mathrm{n}(\%)$. S. pneumoniae: Streptococcus pneumoniae. ${ }^{\#}$ : S. pneumoniae, Haemophilus influenzae, Staphylococcus aureus, H. parainfluenzae and other streptococci; use of PPIs was not associated with causation of CAP by oropharyngeal bacteria ( $p=0.41$ versus nonoropharyngeal and unidentified pathogens).

$\because$ : Escherichia coli and Klebsiella pneumoniae.

pneumonia. Although such a mechanism has been demonstrated in mechanically ventilated patients, it remains speculative in CAP [23-25]. The current study was the first to include elaborate microbial data, acquired using an extensive diagnostic protocol, to identify the causative agent of CAP. As in only two $(2 \%)$ current PPI users' CAP was caused by gastrointestinal bacteria, overgrowth and aspiration of gastrointestinal flora do not seem to be the most prominent cause of pneumonia during acid-suppressive treatment. Our alternative hypothesis was that overgrowth of oropharyngeal bacteria during PPI treatment predisposes patients to developing CAP. Plausibly, clearance of oropharyngeal bacteria is reduced when the $\mathrm{pH}$ of aspirated gastric contents is increased and possibly of the oropharyngeal fluid, as the proton pump is also assumed to be present in the larynx [26-28]. However, the frequency of CAP caused by bacteria that typically colonise the oropharynx was not increased in patients using a PPI. Five $(42 \%)$ cases of pneumonia from the 12 patients recently starting PPI treatment were caused by oropharyngeal bacteria. Thus, in the group in which the risk of CAP was highest, overgrowth of either gastrointestinal or oropharyngeal bacteria does not seem to explain the association between use of PPIs and risk of CAP.

Given our findings that revoke microbial or noncausal pathways as underlying mechanisms of the association, future research should be directed towards other PPI properties or other types of bias. One possible explanation could be the immunomodulatory effects of PPIs. Omeprazole and lansoprazole have been shown to inhibit the expression of adhesion molecules on neutrophils, indicating that PPIs may diminish adequate transmigration of leukocytes to inflammatory sites [29, 30]. In a small study of 10 healthy volunteers, a single oral dose of omeprazole $(40 \mathrm{mg}$ ) decreased reactive oxygen production and neutrophil bactericidal activity [31]. Experimental evidence suggests that omeprazole elevates intralysosomal $\mathrm{pH}$ through inhibition of the neutrophil proton pump, thereby reducing the production of toxic oxidants $[32,33]$. In the present study, we were unable to explore this possible causal pathway.

The major weaknesses of our study are inherent to its observational design. Residual confounding might be present, as we did not have information on the indications for the PPI treatment of all patients, or on medical diagnoses and lifestyle of controls. Instead, we used proxies to identify comorbidities (COPD or asthma, CHF and DM). As shown in figure 1, the proxies for COPD or asthma and DM were very reliable. Remarkably, COPD or asthma were present according to the proxy but not recorded in the chart in $34 \%$ of all cases with COPD or asthma. As only $15 \%$ of cases had COPD or asthma that was not identified by our proxy, it seems that the proxy might even perform better than a)

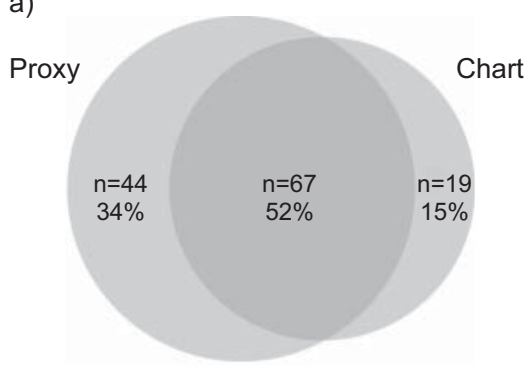

b)

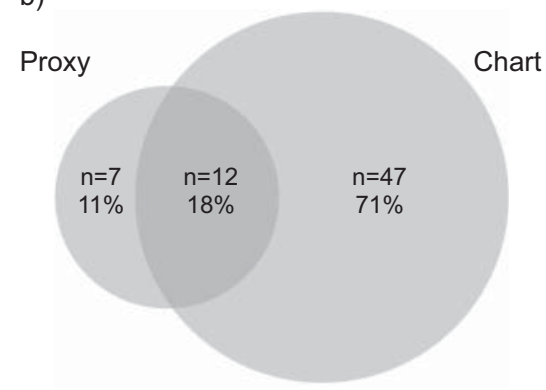

c)

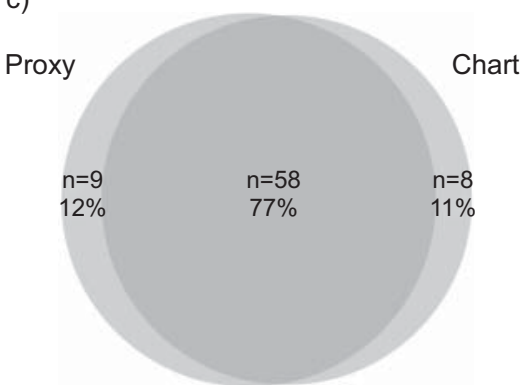

FIGURE 1. Correspondence of proxies to medical chart information for the comorbidities a) chronic obstructive pulmonary disease and asthma, b) congestive heart failure, and c) diabetes mellitus. 
medical record scoring, as it is unlikely that patients would receive and fill two or more prescriptions for airway medication if disease was not present. In the Netherlands, it is possible for a general practitioner to treat a patient with mild COPD or asthma. The proxy used for CHF is less consistent with medical record scoring. However, the number of CHF patients identified by physicians was low; therefore, the impact of the disease as a possible confounder would remain moderate, also with better performance of proxies.

Regarding lifestyle, as no such information was available for the controls, to evaluate the possibility of confounding, we searched for associations between both smoking and alcohol abuse and use of PPI treatment within the pneumonia patients. This analysis showed that cases who smoked were less likely to use PPIs than nonsmoking patients (11 versus $28 \%$, respectively) and that there was no difference for excessive alcohol use versus none (22 versus $23 \%$, respectively). Considering that these habits are risk factors for pneumonia, this could indicate an underestimation of the true association between PPI use and pneumonia in our study.

Another limitation could be the origin of the controls. Instead of hospital controls, we selected population controls. We feel confident that population controls better represent the population from which our cases originated. The PHARMO database hold a very representative sample of the Dutch population and a prior study showed that the patients admitted with pneumonia to the St. Antonius Hospital resemble patients studied in PHARMO [34]. The prevalence of PPI use is comparable for all parts of the Netherlands [35]. Finally, an issue that can only be addressed in a randomised controlled trial is that of poor adherence. Prescriptions for PPIs do not directly reflect exposure to PPIs and patients who are being prescribed a PPI for prophylaxis of gastrointestinal ulcers will adhere less to therapy than patients with active ulcers or dyspepsia. This might be the reason that the risk seems to fade out as PPI therapy turns chronic, because continued use will often reflect prophylactic therapy, whereas short-term use will mainly be indicated in active ulcers.

In conclusion, recent initiation of PPI treatment is associated with an almost three-fold increase in the risk of CAP. Study of the patients recently prescribed PPI treatment showed that the association is not likely to be attributable to protopathic bias. Neither gastrointestinal nor oropharyngeal bacteria were more present in patients using a PPI compared with patients not using a PPI. Given these findings, further study on the causal pathway of the increased risk for pneumonia during PPI use should be directed towards other PPI properties.

\section{STATEMENT OF INTEREST}

None declared.

\section{REFERENCES}

1 Dublin S, Walker RL, Jackson ML, et al. Use of proton pump inhibitors and $\mathrm{H}_{2}$ blockers and risk of pneumonia in older adults: a population-based case-control study. Pharmacoepidemiol Drug Saf 2010; 19: 792-802.

2 Eom CS, Jeon CY, Lim JW, et al. Use of acid-suppressive drugs and risk of pneumonia: a systematic review and meta-analysis. CMAJ 2011; 183: 310-319.
3 Eurich DT, Sadowski CA, Simpson SH, et al. Recurrent community-acquired pneumonia in patients starting acid-suppressing drugs. Am J Med 2010; 123: 47-53.

4 Gau JT, Acharya U, Khan S, et al. Pharmacotherapy and the risk for community-acquired pneumonia. BMC Geriatr 2010; 10: 45.

5 Gulmez SE, Holm A, Frederiksen H, et al. Use of proton pump inhibitors and the risk of community-acquired pneumonia: a population-based case-control study. Arch Intern Med 2007; 167: 950-955.

6 Johnstone J, Nerenberg K, Loeb M. Meta-analysis: proton pump inhibitor use and the risk of community-acquired pneumonia. Aliment Pharmacol Ther 2010; 31: 1165-1177.

7 Laheij RJ, Sturkenboom MC, Hassing RJ, et al. Risk of communityacquired pneumonia and use of gastric acid-suppressive drugs. JAMA 2004; 292: 1955-1960.

8 Myles PR, Hubbard RB, McKeever TM, et al. Risk of communityacquired pneumonia and the use of statins, ace inhibitors and gastric acid suppressants: a population-based case-control study. Pharmacoepidemiol Drug Saf 2009; 18: 269-275.

9 Rodriguez LA, Ruigomez A, Wallander MA, et al. Acid-suppressive drugs and community-acquired pneumonia. Epidemiology 2009; 20: 800-806.

10 Sarkar M, Hennessy S, Yang YX. Proton-pump inhibitor use and the risk for community-acquired pneumonia. Ann Intern Med 2008; 149: 391-398.

11 Gregor JC. Acid suppression and pneumonia: a clinical indication for rational prescribing. JAMA 2004; 292: 2012-2013.

12 Sataloff RT. Community-acquired pneumonia and use of gastric acid-suppressive drugs. JAMA 2005; 293: 795-796.

13 Vakil N. Acid inhibition and infections outside the gastrointestinal tract. Am J Gastroenterol 2009; 104: Suppl. 2, S17-S20.

14 Penning-van BF, van Herk-Sukel M, Gale R, et al. Three-year dispensing patterns with long-acting inhaled drugs in COPD: a database analysis. Respir Med 2011; 105: 259-265.

15 The PHARMO Institute. www.pharmo.nl. Date last accessed: January 2011.

16 Endeman H, Schelfhout V, Voorn GP, et al. Clinical features predicting failure of pathogen identification in patients with community acquired pneumonia. Scand J Infect Dis 2008; 40: 715-720.

17 St Antonius Hospital. Dexamethasone Infusion in Communityacquired Pneumonia (Ovidius). NCT0047164. www.clinicaltrials. gov/ct2/show /NCT00471640?term $=$ NCT00471640\&rank=1 Date last accessed: July 8, 2011. Date last updated: September 24, 2010.

18 Fine MJ, Auble TE, Yealy DM, et al. A prediction rule to identify low-risk patients with community-acquired pneumonia. $N$ Engl J Med 1997; 336: 243-250.

19 Van Mens SP, Meijvis SC, Endeman H, et al. Longitudinal analysis of pneumococcal antibodies during community-acquired pneumonia reveals a much higher involvement of Streptococcus pneumoniae than estimated by conventional methods alone. Clin Vaccine Immunol 2011; 18: 796-801.

20 WHO Collaborating Center for Drug Statistics Methodology. Guidelines for ATC Classification and DDD Assignment. Oslo, World Health Organization, 2000.

21 Almirall J, Bolibar I, Serra-Prat $\mathrm{M}$, et al. Inhaled drugs as risk factors for community-acquired pneumonia. Eur Respir J 2010; 36: 1080-1087.

22 Crim C, Calverley PM, Anderson JA, et al. Pneumonia risk in COPD patients receiving inhaled corticosteroids alone or in combination: TORCH study results. Eur Respir J 2009; 34: 641-647.

23 Inglis TJ, Sherratt MJ, Sproat LJ, et al. Gastroduodenal dysfunction and bacterial colonisation of the ventilated lung. Lancet 1993; 341: 911-913.

24 Messori A, Trippoli S, Vaiani M, et al. Bleeding and pneumonia in intensive care patients given ranitidine and sucralfate for prevention of stress ulcer: meta-analysis of randomised controlled trials. BMJ 2000; 321: 1103-1106. 
25 Prod'hom G, Leuenberger P, Koerfer J, et al. Nosocomial pneumonia in mechanically ventilated patients receiving antacid, ranitidine, or sucralfate as prophylaxis for stress ulcer. A randomized controlled trial. Ann Intern Med 1994; 120: 653-662.

26 Altman KW, Haines GK III, Hammer ND, et al. The $\mathrm{H}^{+} / \mathrm{K}^{+}$ATPase (proton) pump is expressed in human laryngeal submucosal glands. Laryngoscope 2003; 113: 1927-1930.

27 Altman KW, Waltonen JD, Hammer ND, et al. Proton pump $\left(\mathrm{H}^{+} /\right.$ $\mathrm{K}^{+}$-ATPase) expression in human laryngeal seromucinous glands. Otolaryngol Head Neck Surg 2005; 133: 718-724.

28 Altman KW, Waltonen JD, Tarjan G, et al. Human lung mucous glands manifest evidence of the $\mathrm{H}^{+} / \mathrm{K}^{+}$-ATPase proton pump. Ann Otol Rhinol Laryngol 2007; 116: 229-234.

29 Ohara T, Arakawa T. Lansoprazole decreases peripheral blood monocytes and intercellular adhesion molecule-1-positive mononuclear cells. Dig Dis Sci 1999; 44: 1710-1715.

30 Yoshida N, Yoshikawa T, Tanaka Y, et al. A new mechanism for anti-inflammatory actions of proton pump inhibitors-inhibitory effects on neutrophil-endothelial cell interactions. Aliment Pharmacol Ther 2000; 14: Suppl. 1, 74-81.
31 Zedtwitz-Liebenstein K, Wenisch C, Patruta S, et al. Omeprazole treatment diminishes intra- and extracellular neutrophil reactive oxygen production and bactericidal activity. Crit Care Med 2002; 30: 1118-1122.

32 Agastya G, West BC, Callahan JM. Omeprazole inhibits phagocytosis and acidification of phagolysosomes of normal human neutrophils in vitro. Immunopharmacol Immunotoxicol 2000; 22: 357-372.

33 Suzuki M, Mori M, Miura S, et al. Omeprazole attenuates oxygenderived free radical production from human neutrophils. Free Radic Biol Med 1996; 21: 727-731.

34 van de Garde EM, Endeman H, van Hemert RN, et al. Prior outpatient antibiotic use as predictor for microbial aetiology of community-acquired pneumonia: hospital-based study. Eur J Clin Pharmacol 2008; 64: 405-410.

35 National Institute of Public Health and the Environment. Gebruik Maagmiddelen het Hoogst in de Regio Hart voor Brabant [Use of gastrointestinal agents is highest on the Hart voor Brabant region] www.zorgatlas.nl/zorg/genees-en-hulpmiddelen/geneesmiddelengebruik/standaarddagdosering-maagmiddelen/\#breadcrumb Date last accessed: August 5, 2011. Date last updated: June 30, 2011. 